\title{
Critical spectral statistics in two-dimensional interacting disordered systems
}

\author{
E. Cuevas \\ Departamento de Física, Universidad de Murcia, E-30071 Murcia, Spain.
}

(February 13, 2018)

\begin{abstract}
The effect of Coulomb and short-range interactions on the spectral properties of two-dimensional disordered systems with two spinless fermions is investigated by numerical scaling techniques. The size independent universality of the critical nearest level-spacing distribution $P(s)$ allows one to find a delocalization transition at a critical disorder $W_{\mathrm{c}}$ for any non-zero value of the interaction strength. At the critical point the spacings distribution has a small-s behavior $P_{c}(s) \propto s$, and a Poisson-like decay at large spacings.
\end{abstract}

PACS number(s): 71.30, 72.15 Rn, 71.55 Jv

It has been shown that the statistical properties of spectra of disordered one-electron systems are closely related to the localization properties of the corresponding wave functions [1] 3]. In the localized regime, states close in energy have an exponentially small overlap and their levels are uncorrelated. So the corresponding normalized spacings $s$ are distributed according to the Poisson law

$$
P_{\mathrm{P}}(s)=\exp (-s) \text {. }
$$

On the other hand, in the metallic regime the large overlap of delocalized states induces correlations in the spectrum leading to the well known level repulsion effect. If the system is invariant under rotations and under time-reversal symmetry the spacings follow WignerDyson statistics

$$
P_{\mathrm{W}}(s)=\frac{\pi}{2} s \exp \left(-\frac{\pi}{4} s^{2}\right) .
$$

The two limiting behaviors given by Eqs. (1) and (2) correspond to an infinite system. In Refs. [2, 14 was found that a third, size independent distribution, appears at the metal-insulator transition (MIT) in the three dimensional (3D) Anderson model. This behavior is the basis of a different method to detect the MIT whose advantage resides in that no knowledge of the eigenfunctions is needed. We will extend this procedure to two-electron interacting disordered systems.

The study of the energy spectrum has already provided important results about the nature of the states and about the transport regimes that one can expect. In fact, the method has been successful in the location of the MIT in the 3D Anderson model [2, 5 , 8], in two dimensional (2D) disordered systems with symplectic symmetry [9 11], in the determination of the critical interaction strength in many-body systems 12 14, and in the study of transport regimes in the 2D Anderson model [15.16.

As dimension two is marginal, one can expect a interaction-driven delocalization transition in a $2 \mathrm{D}$ interacting disordered system because of the following reasons. First of all, the scaling theory of localization including the combined effects of disorder and interactions predicts that a $2 \mathrm{D}$ system may remain metallic even in the limit of zero temperature 17, 18. Secondly, many recent experimental results have presented strong evidence for a delocalization transition in 2D systems without magnetic scattering effects employing different materials and designs [19 25]. All these experiments show clear indications that strong electron-electron interactions partially suppress the quantum interference effects responsible for localization. Furthermore, a recent numerical study of two-interacting bosons in $2 \mathrm{D}$ by means of their decay length in long bars [26] showed evidence for a localized to extended states transition.

In this Letter we study the level statistics of two interacting electrons in a $2 \mathrm{D}$ random potential. We consider both long-range Coulomb interactions and short-range interactions and calculate numerically the correlations in the exact two-electron spectrum. Our main result is that the nearest level-spacing distribution $P(s)$ is universal in the critical region showing a delocalization transition for any finite value of the interaction strength.

We consider spinless fermions on a sample of size $L \times L$ described by the standard Anderson-Hubbard Hamiltonian

$$
\begin{aligned}
H= & t \sum_{i, j}\left(a_{i, j+1}^{\dagger} a_{i, j}+a_{i+1, j}^{\dagger} a_{i, j}+\text { h.c. }\right) \\
& +\sum_{i, j} \epsilon_{i, j} a_{i, j}^{\dagger} a_{i, j}+H_{\mathrm{int}},
\end{aligned}
$$

where the operator $a_{i, j}^{\dagger}\left(a_{i, j}\right)$ creates (destroys) an electron at site $(i, j)$ of a square lattice and $\epsilon_{i, j}$ is the energy of this site chosen randomly between $(-W / 2, W / 2)$ with uniform probability. The hopping matrix element $t$ is taken equal to -1 and the lattice constant equal to 1 , which sets the energy and length scales, respectively. For the long-range case, the interaction Hamiltonian is given by $[12]$

$$
H_{\mathrm{int}}=U \sum_{i, j>k, l} \frac{a_{i, j}^{\dagger} a_{i, j} a_{k, l}^{\dagger} a_{k, l}}{\left|\boldsymbol{r}_{i, j}-\boldsymbol{r}_{k, l}\right|}
$$

while for the short-range case we choose a nearestneighbor interaction Hamiltonian 


$$
\begin{aligned}
H_{\mathrm{int}}= & U \sum_{i, j}\left(a_{i, j}^{\dagger} a_{i, j} a_{i, j+1}^{\dagger} a_{i, j+1}\right. \\
& \left.+a_{i, j}^{\dagger} a_{i, j} a_{i+1, j}^{\dagger} a_{i+1, j}\right) .
\end{aligned}
$$

In both cases, in order to reduce edge effects, we use periodic boundary conditions, i.e., $a_{L+1, j}^{\dagger}=a_{1, j}^{\dagger}, a_{i, L+1}^{\dagger}=$ $a_{i, 1}^{\dagger}, a_{0, j}^{\dagger}=a_{L, j}^{\dagger}, a_{i, 0}^{\dagger}=a_{i, L}^{\dagger}$.

The $L^{4}$ eigenstates of the Hamiltonian (3) are either symmetric or antisymmetric with respect to the interchange of the electron positions. We restrict our investigation to the two-electron Hilbert subspace spanned in the basis of $N=L^{2}\left(L^{2}-1\right) / 2$ antisymmetric products of one-electron states

$$
\left|\psi_{i, j ; k, l}\right\rangle=\frac{1}{\sqrt{2}}\left(a_{i, j}^{\dagger} a_{k, l}^{\dagger}-a_{k, l}^{\dagger} a_{i, j}^{\dagger}\right)|0\rangle,
$$

where $|0\rangle$ is the vacuum state. The resulting $N \times N$ Hamiltonian matrix is numerically diagonalized using techniques for large sparse matrices, in particular a Lanczos tridiagonalization without reorthogonalization method [27]. The strength $U$ of the interactions is varied between $0-10$ and the disorder strength ranges the interval $(0.2,16)$. The system size varies from $L=6$ to 20 and the number of random realizations is such that for a given triad of $\{U, L, W\}$ the number of studied eigenvalues in the range $(-1,1)$ was kept around $2.5 \times 10^{4}$.

In order to minimize fluctuations in $P(s)$, we used in the calculations for $U \gtrsim 1$ the levels contained in the interval $(-1,1)$ around the band center. Although this energy window exceeds the one-electron level spacing $\Delta_{1}$, does not introduce hidden correlations (unlike for the non-interacting case $U=0$ or for $U \ll 1$ ) in the twoelectron spectrum because of the strong mixing of the states directly coupled by the interaction. In Ref. 28] was shown, modeling the full Hamiltonian (3) by a Gaussian ensemble of random matrices with a preferential basis [29, 30, that the spread width $\Gamma_{2}$ of a two-electron state is of the order of $\Delta_{1}$. In any case, we verified that, within the statistical errors, there is no significant changes in $P(s)$ if we used an energy window $\Delta_{\epsilon}<\Delta_{1}$.

To quantitatively analyze the scaling of the statistical properties of the eigenvalue spectrum one can use one of the two following variables widely employed. The first one [2] 12 14] is given by $\eta=\int_{s_{0}}^{\infty}[P(s)-$ $\left.P_{\mathrm{W}}(s)\right] d s / \int_{s_{0}}^{\infty}\left[P_{\mathrm{P}}(s)-P_{\mathrm{W}}(s)\right] d s$, where for $s_{0}$ one can choose one of the two crossing points of the curves $P_{\mathrm{P}}(s)$ and $P_{\mathrm{W}}(s)$, namely $s_{0}=0.473$ and $s_{0}=2.002$. The former is representative of the small-s part of $P(s)$, whereas the latter is representative for its tail. The second variable [5, 6, 31], based in the $\Delta_{3}$ statistics, which take into account correlations between non adjacent levels, is given by $\eta=\frac{1}{30} \int_{0}^{30} \Delta_{3}(L) d L$. In this Letter we use a different scaling variable [16, 32 based on the variance of $P(s)$, since it contains information about the whole distribution, namely

$$
\eta(L, W)=\frac{\operatorname{var}(s)-0.273}{1-0.273},
$$

which describes the relative deviation of $\operatorname{var}(s)$ from the Wigner-Dyson limit due to the finiteness of the system. In Eq. (而) $\operatorname{var}(s)=\left\langle s^{2}\right\rangle-\langle s\rangle^{2}$, and 0.273 and 1 are the variances of Wigner-Dyson and Poisson distributions, respectively. In this way, $\eta(L, W)$ ranges from $0[P(s)=$ $\left.P_{\mathrm{W}}\right]$ to $1\left[P(s)=P_{\mathrm{P}}\right]$. We have checked that our results are basically independent of which of the three definitions of $\eta$ mentioned is used, although the results based on Eq. (7) are more accurate.

Figure 1 shows the disorder dependence of $\eta$ for a longrange interaction with $U=1$ for different system sizes: $L=6(\circ), 9(\square), 16(\diamond)$ and $20(\nabla)$. One can distinguish three different regions, in which $\eta$ increases, remain constant, or decreases with increasing $L$. The curves for different sizes cross at a common point, $W_{c}$, which corresponds to the interaction-induced delocalization transition.

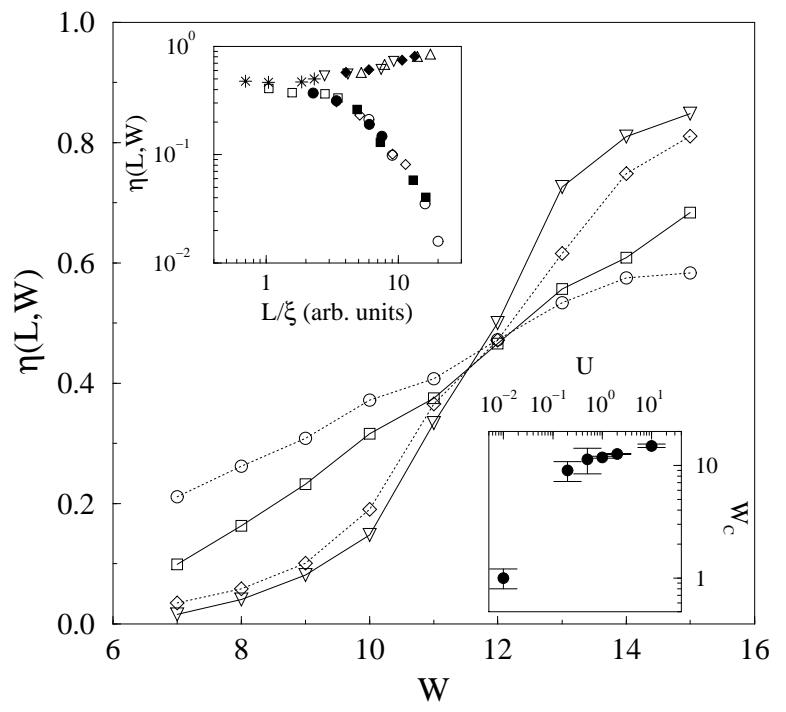

FIG. 1. Scaling variable $\eta$ as a function of $W$ for a long-range interaction with $U=1$. System sizes: $L=6$ (०), $9(\square), 16(\diamond)$ and $20(\nabla)$. The critical disorder is given by the point for which $\eta$ is independent of $L$. Upper inset: Verification of the one-parameter scaling assumption for $\eta(L, W)$. The horizontal scale is arbitrary. Lower inset: $U$ dependence of $W_{c}$.

In the following we check whether $\eta(L, W)$ can be expressed by a one-parameter scaling function

$$
\eta(L, W)=f(L / \xi(W)),
$$

where the scaling parameter $\xi$ is the two-electron localization length in the localized regime, and the two-particle correlation length in the extended regime. To perform this scaling procedure with the $\eta$ data, the range of $\eta$ values for different $L$ at any given disorder $W_{1}$ must overlap the range of $\eta$ values for various $L$ for at least one 
different disorder $W_{2}$. Equation (8) implies that in a loglog plot of $\eta(L, W)$ versus $L$ all data should collapse in a common curve when translated by an amount $\ln \xi(W)$ along the horizontal axis. This curve has a single branch when there is no transition, while it develops two separate branches when a transition is present. In the upper inset of Fig. 1, we re-plot the data of Fig. 1 by rescaling $L$. Note that one can fit most of the points onto a common curve with two branches, one growing $\left(W>W_{c}\right)$ and another decaying $\left(W<W_{c}\right)$. This results confirm the scaling hypothesis for $\eta(L, W)$ and thus we have found the function $\xi(W)$ up to an arbitrary factor.

Next we determine the critical disorder $W_{c}$ and the critical exponent $\nu$. For this purpose we used the singularity of the correlation length near $W_{c}$

$$
\xi(W)=\xi_{0}\left|W-W_{c}\right|^{-\nu},
$$

where $\xi_{0}$ is a constant. Using Eq. (8) and taking into account that $\eta(L, W)$ is analytical for a finite system, we can write around the critical point

$$
\eta(L, W)=\eta_{c}+\sum_{n} A_{n}\left(W-W_{c}\right)^{n} L^{n / \nu},
$$

from which one can extract $W_{c}$ and $\nu$. In practice, we have truncated the series at $n=4$. We performed an statistical analysis of the data in the range $10 \leq W \leq$ 14 with the Levenberg-Marquardt method for nonlinear least-squares models. The most likely fit is determined by minimizing the $\chi^{2}$ statistic of the fitting function (10). The critical disorder found is $W_{\mathrm{c}}=11.8 \pm 0.2$, and the corresponding critical exponent is equal to $\nu=1.2 \pm 0.2$.

We repeated the same kind of calculations for different values of the interaction strength $U$ including the noninteracting case $(U=0)$. For $U \ll 1$ we obtained $\eta$ using Eq. (7) in a energy window $\Delta_{\epsilon}<\Delta_{1}$. The $U$ dependence of the critical disorder $W_{c}$ is shown in the lower inset of Fig. 1. The $W_{c}$ found increases monotonously with $U$ starting from very small values. In the non-interacting limit there is no critical behavior and all states are localized, as expected.

We have also analyzed the behavior of two electrons subjected to a short-range interaction, Eq. (5). The corresponding results are depicted if Fig. 2. The system sizes used are the same as those appearing in Fig. 1. For $U=1$ the critical disorder and exponent found are $W_{c}=10.4 \pm 0.2$ and $1.3 \pm 0.2$, respectively. The dependence of $W_{c}$ on $U$, shown in the lower inset of Fig. 2 , is slightly different than that found for the long-range case. $W_{c}$ increases with $U$ and reaches a saturation value around $U \approx 2$. After saturation $W_{c}$ decreases for the largest value of the interaction strength used, $U=10$. The reason is that for this value of $U$, as we have checked, the interaction slightly splits the two-interacting particle band into lower and upper Hubbard bands.

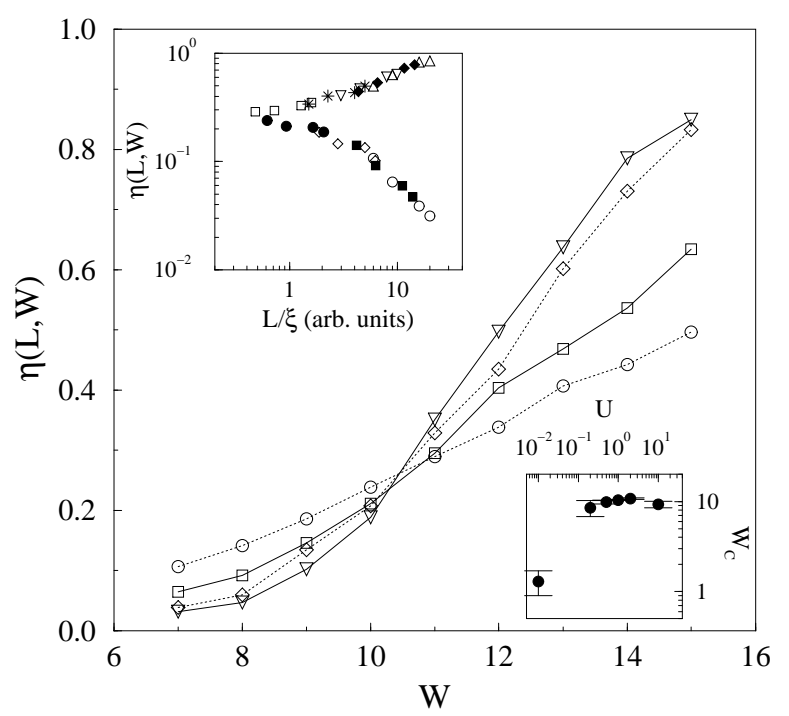

FIG. 2. Same as in Fig. 1 for a short-range interaction with $U=1$.

Finally we focus on the level statistics at the critical point. For the orthogonal symmetry $P_{c}(s) \propto s$ as $s \rightarrow 0$ [2,33], while for $s \gg 1$, two different analytical expression were proposed. One of them 2, 2, assumes that $P_{c}(s)$ is Poissonian and the other [34 has the asymptotic form $P_{c}(s) \propto \exp \left(-A_{c} s^{\alpha}\right)$, where $\alpha$ is related to the critical exponent $\nu$ and to the dimensionality $d$ through $\alpha=1+(d \nu)^{-1}$. In order to diminish the magnitude of the relative fluctuations and to analyze the asymptotic behavior in detail, it is more convenient to consider the cumulative level spacing distribution function $I(s)=\int_{s}^{\infty} P\left(s^{\prime}\right) d s^{\prime}$. The Poisson distribution Eq. (11) and the Wigner surmise Eq. (2) yield $I_{\mathrm{P}}(s)=\exp (-s)$ and $I_{\mathrm{W}}(s)=\exp \left(-\pi s^{2} / 4\right)$, respectively.

We have checked that for various $L$ considered at the critical disorder $W_{c}$ for a given $U$, the best fit using a $\chi^{2}$ criterion in the interval $2<s<5$ yields $\alpha \approx 1.0 \pm 0.1$ and, therefore, $P_{c}(s)$ at large $s$ is very close to a Poissonian decay, thus confirming the ideas of [2, 胞. A similar conclusion was obtained in Ref. [8] in the Anderson transition using very large system sizes. The results for the numerical calculations of the critical $I_{c}(s)$ for a long-range interaction with $U=1$ are shown in Fig. 3 for $L=12$ $(\circ), 16(\square)$ and $20(\diamond)$. Note that the critical $I_{c}(s)$ is a $L$-independent universal scale-invariant function that interpolates between Wigner and Poisson limits. Solid and dashed lines correspond to $I_{\mathrm{P}}(s)$ and $I_{\mathrm{W}}(s)$, respectively. In the upper inset we display our results for $I_{c}(s)$ in the limit $s \gg 1$. One clearly see that the Poissonian tail is recovered for large spacings. This behavior is similar to that in the insulating regime, although the decay rate $A_{c}$ is larger than unity. The straight line fitting the data in the interval $2<s<5$ is $\ln I_{c}(s)=-1.32 s+0.33$. In the lower inset we show the limiting behavior of $P_{c}(s)$ as $s \rightarrow 0$. Here we find that $P_{c}(s) \propto s$ according with 
predictions of Refs. [2,33]. The straight line fitting the data in the interval $4 \times 10^{-3}<s<10^{-1}$ is $P_{c}(s)=3 s$.

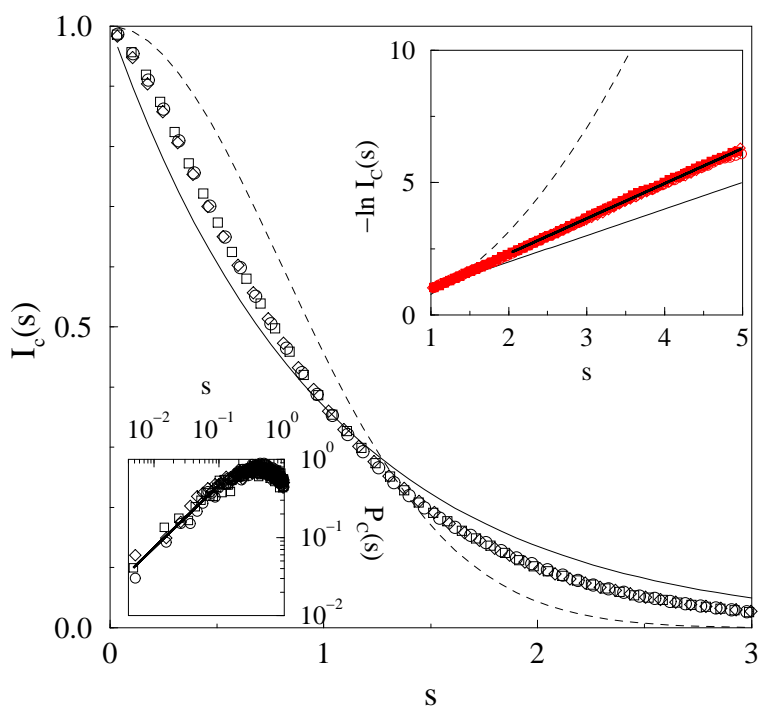

FIG. 3. Integrated probability $I_{c}(s)$ at the critical disorder for a long-range interaction with $U=1$. System size: $L=12$ $(\circ), 16(\square), 20(\diamond)$. Solid and dashed lines are $I_{\mathrm{P}}(s)$ and $I_{\mathrm{W}}(s)$, respectively. Upper inset: Large- $s$ part of $I_{c}(s)$; the fitted line is $\ln I_{c}(s)=-1.32 s+0.33$. Lower inset: double logarithmic plot of $P_{c}(s)$ for $s \ll 1$; the fitted line is $P_{c}(s)=3 s$.

To summarize, we have investigated the energy levels statistics of a two-electron disordered $2 \mathrm{D}$ system with a long-range or a short-range interaction. In both cases, the scaling function was calculated using the statistics of the nearest-neighbor eigenvalues of the two-electron states, that have been obtained numerically. By performing a finite size scaling analysis we found a critical disorder $W_{c}$, for any non-zero value of the interaction strength $U$. When $U \rightarrow 0, W_{c} \rightarrow 0$ and so we recover the expected results of the non-interacting system where all the states are localized. $P_{c}(s)$ is linear for small $s$ while the large-s part of $P_{c}(s)$ obtained is shown to have a Poisson-like decay.

We would like to thank M. Ortuño and D. Weinmann for useful discussions. The Spanish DGES, project number PB96-1118, is also acknowledge for financial support.

[1] B.L. Altshuler and B.I. Shklovskii, Zh. Eksp. Teor. Fiz. 91, 220 (1986) [Sov. Phys. JETP 64, 127 (1986)].

[2] B.I. Shklovskii, B. Shapiro, B.R. Sears, P. Lambrianides, and H.B. Shore, Phys. Rev. B 47, 11487 (1993).

[3] V.E. Kravtsov, I.V. Lerner, B.L. Altshuler and A.G. Aronov, Phys. Rev. Lett. 72, 888 (1994).
[4] B.L. Altshuler, I.Kh. Zharekeshev, S.A. Kotochigova, and B.I. Shklovskii, Zh. Eksp. Teor. Fiz. 94, 343 (1988) [Sov. Phys. JETP 67, 25 (1988)].

[5] E. Hofstteter and M. Schreiber, Phys. Rev. B 49, 14726 (1994).

[6] E. Hofstteter, Phys. Rev. B 57, 12763 (1998).

[7] I.Kh. Zharekeshev and B. Kramer, Phys. Rev. B 51, 17239 (1995).

[8] I.Kh. Zharekeshev and B. Kramer, Phys. Rev. Lett. 79, 717 (1997).

[9] S.N. Evangelou, Phys. Rev. Lett. 75, 2550 (1995).

[10] L. Schweitzer and Kh. Zharekeshev, J. Phys.: Con. Mat. 7, L377 (1995).

[11] L. Schweitzer and Kh. Zharekeshev, J. Phys.: Con. Mat. 9, L441 (1997).

[12] R. Berkovits and Y. Avishai, Phys. Rev. Lett. 80, 568 (1998).

[13] Ph. Jacquod, Phys. Stat. Sol. (b) 205, 263 (1998).

[14] Ph. Jacquod and D.M. Shepelyansky, Phys. Rev. Lett. 79, 1837 (1997).

[15] Kh. Zharekeshev, M. Batsch, and B. Kramer, Europhys. Lett. 34, 587 (1996).

[16] E. Cuevas, M. Ortuño, J. Ruiz, E. Louis, and J.A. Vergés, J. Phys.: Con. Mat. 10, 295 (1998).

[17] A.M. Finkelstein, Zh. Eksp. Teor. Fiz. 84, 168 (1983) [Sov. Phys. JETP 57, 97 (1983)]; -, Z. Phys. A 56, 189 (1984).

[18] C. Castellani, C. Di Castro and P.A. Lee, Phys. Rev. B 57, R9381 (1998).

[19] S.V. Kravchenko, G.V. Kravchenko, J.E. Furneaux, V.M. Pudalov and M. D'Iorio, Phys. Rev. B 50, 8039 (1994).

[20] S.V. Kravchenko, V.E. Mason, G.E. Bowker, J.E. Furneaux, V.M. Pudalov and M. D'Iorio, Phys. Rev. B 51, 7038 (1995).

[21] S.V. Kravchenko, D. Simonian, M.P. Sarachik, W. Mason and J.E. Furneaux, Phys. Rev. Lett. 77, 4938 (1996).

[22] D. Popović, A.B. Fowler and S. Washburn, Phys. Rev. Lett. 79, 1543 (1997).

[23] D. Popović and S. Washburn, Phys. Rev. B 56, R10048 (1997).

[24] M.Y. Simmons, et al., Phys. Rev. Lett. 80, 1292 (1998).

[25] Y. Hanein, et al., Phys. Rev. Lett. 80, 1288 (1998).

[26] M. Ortuño and E. Cuevas, cond-mat/9808104.

[27] J.K. Cullum and R.A. Willoughby, Lanczos Algorithms for Large Symmetric Eigenvalue Computations (Birkhauser, Basel, 1985).

[28] D. Weinmann, J.-L. Pichard, and Y. Imry, J. Phys. I France 7, 1559 (1997).

[29] D. Weinmann, and J.-L. Pichard, Phys. Rev. Lett. 77, 1556 (1996).

[30] J.-L. Pichard, and B. Shapiro, J. Phys. I France 4, 623 (1994).

[31] E. Hofstteter and M. Schreiber, Phys. Rev. B 48, 16979 (1993).

[32] E. Cuevas, E. Louis, and J.A. Vergés, Phys. Rev. Lett. 77, 1970 (1996).

[33] M.L. Mehta, Random Matrices (Academic Press, Boston, 1991).

[34] A.G. Aronov, V.E. Kravtsov, and I.V. Lerner, Pis'ma Zh. Eksp. Teor. Fiz. 59, 39 (1994) [JETP Lett. 59, 40 (1994)]; -, Phys. Rev. Lett. 74, 1174 (1995);V.E. Kravtsov and 
I.V. Lerner, J. Phys. A, 28, 3623 (1995). 